\title{
Desenvolvimento de uma Bengala Automatizada Utilizando Arduino para Deficientes Visuais
}

\author{
Rayssa C. Costa ${ }^{1}$, Mayara Volkmer ${ }^{1}$, Simone S. F. Souza ${ }^{2}$, Fernando P. A. Lima ${ }^{1}$ \\ ${ }^{1}$ Instituto Federal do Mato Grosso (IFMT), Campus Avançado Tangará da Serra, \\ Rua José de Oliveira (28), 980N, Vila Horizonte, 78300-000, Tangará da Serra, MT \\ ${ }^{2}$ Universidade do Estado de Mato Grosso (UNEMAT), Campus Tangará da Serra, \\ Rodovia MT - 358 (Avenida Inácio Bittencourt Cardoso), Km 07 (s/n), Jardim \\ Aeroporto, 78300-000, Tangará da Serra, MT \\ 1 \{rayssaaccegmail.com, mayaracaroline.volkmer@gmail.com, \\ fernando.lima@tga.ifmt.edu.br\}, 2 \{simonefrutuoso.mat@gmail.com\}
}

\begin{abstract}
Resumo. Neste artigo apresenta-se o processo de desenvolvimento, construção e testes de um protótipo de uma bengala automatizada para deficientes visuais. O projeto foi idealizado com o intuito de criar uma ferramenta automatizada para auxiliar os deficientes visuais nas suas atividades diárias, de modo a identificar obstáculos e evitar colisões. Para a montagem do protótipo modelo, foram utilizados materiais de baixo custo e arduino. Durante a elaboração do protótipo, foi realizada uma pesquisa de campo no Centro Municipal de Educação Especial Professora. Isoldi Storck na cidade de Tangará da Serra-MT, onde quatro alunos cegos foram entrevistados e realizaram testes com o protótipo. Através da participação e avaliação dos deficientes, pode-se destacar que os resultados obtidos são satisfatórios, e o protótipo desenvolvido neste trabalho além de seguro e confiável, atende as necessidades identificando obstáculos com precisão.
\end{abstract}

\section{Introdução}

Nos dias atuais, observa-se um grande avanço da tecnologia aplicada objetivando a acessibilidade para deficientes, sejam deficientes físicos, mentais, ou com algum tipo de disfunção. A acessibilidade significa dar a essas pessoas condições para alcançarem e utilizarem, com segurança e autonomia, os espaços, mobiliários e equipamentos urbanos, as edificações, os transportes e os sistemas e meios de comunicação. Neste contexto, este artigo irá abordar um estudo sobre a tecnologia aplicada na acessibilidade para deficientes visuais.

Assim, visualizando o histórico desta deficiência, é possível perceber que a mesma apresentou crescimento em seus índices de ocorrências no passado, pois, de acordo com dados divulgados pela OMS (Organização Mundial da Saúde), na população mundial em 1990, existiam 38 milhões de pessoas cegas e 110 milhões com baixa visão. Já em 1996 o número de cegos subiu para 45 milhões, e 135 milhões de pessoas portadora de baixa visão [Temporin, Kara-Jose 2004].

Entretanto, nos dias atuais, o número de pessoas com cegueira em todo o mundo, diminuiu significativamente em relação aos anos anteriores, graças ao desenvolvimento tecnológico ocorrido no âmbito da saúde e a melhoria nas condições de vida 
populacional. Isso é evidenciado em pesquisa realizada pela revista médica The Lancet Global Health, na qual no ano de 2017 levantou que a cegueira afeta 36 milhões de pessoas da população mundial. Porém, a perspectiva futura de proliferação desta patologia é de aumento novamente, dado que, a revista citada anteriormente, apresenta estimativas de que até 115 milhões de pessoas em todo o mundo possuam cegueira até 2050 .

O IBGE (Instituto Brasileiro de Geografia e Estatística), mostra que, no censo demográfico realizado no ano de 2010, o Brasil possuía cerca de 6,5 milhões de pessoas com alguma deficiência visual, onde 506,377 mil pessoas eram cegas e 6.056.533 milhões possuíam baixa visão.

Segundo Santos et al. (2010), a deficiência visual como a perda irreversível da visão, causadas por hereditariedade ou congênitas, mesmo com o auxílio de óculos e tratamentos clínicos. A mesma pode se subdivide entre severa, moderada ou profunda. Todavia, indivíduos que possuem essa deficiência, desenvolvem de maneira mais eficiente o uso de outros sentidos, como a audição.

A tabela Snellen é um diagrama utilizado para avaliar a acuidade visual de determinado indivíduo [Teixeira 2018], [Florio 2018]. Para ser considerado um portador de deficiência visual, o indivíduo deve apresentar uma capacidade mínima de percepção igual ou menor à 20/200 na tabela Snellen em seu melhor olho ou apresentar campo visual inferior a $20^{\circ}$ na referida tabela [Brasília DF 2013, p.13]. De acordo com a OMS, a cegueira ocorre quando se nota que a percepção visual de uma pessoa é menor que 3/60 em seu melhor olho, depois de feito a correção óptica. Já baixa visão é decorrente quando a percepção visual é inferior a 6/60 no melhor olho do indivíduo em questão [Ramke, Gilbert 2017].

Conforme destacado por Almeida et al. (2016), o Brasil ainda sofre com a precarização de acessibilidade, mesmo possuindo um número elevado de deficientes visuais. Tal fato prejudica em demasia a vida de pessoas portadoras de tal deficiência, de modo que estes indivíduos ficam sujeitos a sofrer com discriminação, desemprego, falta de transporte especial, ausência de uma educação especializada, dentre outros problemas.

Durante a elaboração deste artigo, foi realizada uma pesquisa de campo com alguns deficientes visuais em uma instituição na cidade, visando obter informações acerca das principais dificuldades enfrentadas no dia-a-dia de um deficiente. Quando questionados, os deficientes relataram unanimemente a falta de acessibilidade na cidade, destacando que algo simples como: falta ou mal planejamento de pistas táteis em órgãos públicos; veículos, mesas e cadeiras de lanchonetes nas calçadas; animais soltos na rua; toldos baixos em locais públicos e privados; placas; caçambas de lixo; dificultam a locomoção, e podem ocasionar acidentes.

Corroborando com o que foi relatado, a pesquisa realizada por Bueno (2010), afirma que o SUS (Sistema Único de Saúde), apresenta um número considerável de internações realizadas por deficientes visuais em hospitais públicos em todo o Brasil, onde, na maioria dos casos, essas pessoas se machucam ao bater em algum objeto presente em calçadas ou até em carros, e outro notável número de pacientes realizam entradas por ferimentos na região da cabeça. 
Comumente, deficientes visuais sempre possuem em mãos bengalas que os auxiliam em sua locomoção, utilizando-as à frente do corpo a fim de que está toque nos obstáculos à sua frente antes que os mesmos colidam com seus corpos. Todavia, diante dos fatos expostos, é possível concluir que as bengalas simples atualmente utilizadas pelos cegos não são de todo eficazes, deixando a desejar em diversos aspectos, visto que ainda colocam em risco a saúde dos indivíduos que as utilizam, por conta de não conseguirem identificar objetos acima da linha da cintura e afins, como exemplifica a Figura 1.

Um método utilizado para tentar minimizar as dificuldades enfrentadas por cegos e os auxiliarem na locomoção, foi a criação da Lei $\mathrm{n}^{\circ} 11.126$, em 2005, que regulamenta o uso do cão-guia para deficientes visuais. No entanto, tal recurso não se apresenta totalmente viável, posto que, de acordo com Silva (2018), o cão guia não é permitido em todos os locais, além de que, segundo Bueno (2010), no Brasil, o governo disponibiliza apenas 50 cães gratuitamente adestrados para a população cega.

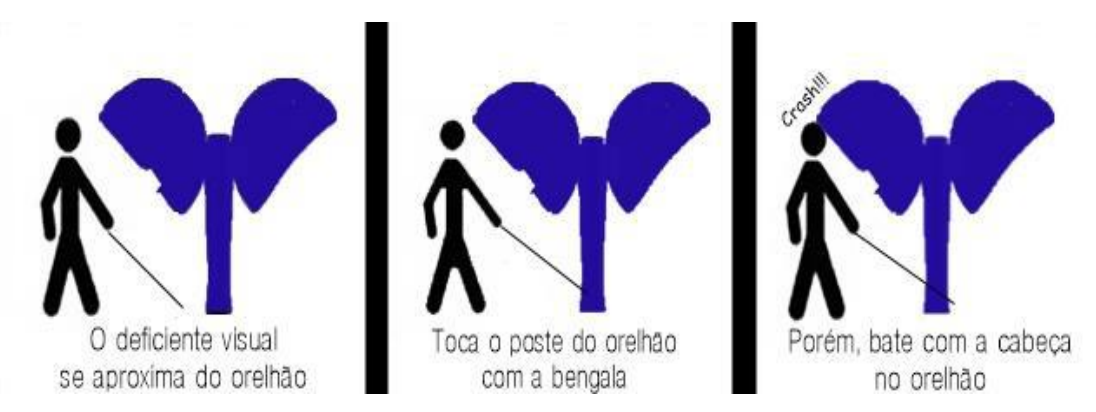

Figura 1. Problemas apresentados em bengalas básicas.

Visando desenvolver tecnologias que propiciem a melhora do estilo de vida de deficientes visuais, foi criada a TA (Tecnologia Assistiva), que de acordo com Pinto (2018), é definida como uma área do conhecimento capaz de dispor de recursos, metodologias e estratégias voltadas para a criação de meios que promovam autonomia e qualidade de vida a pessoas com deficiência, a fim de que estas disponham de maior inclusão social em diversas áreas de necessidades pessoais, como comunicação, mobilidade, alimentação, lazer, educação, dentre outras.

Os autores Bersche e Tonolli (2006), afirmam que a TA pode ser dividida em duas partes, uma de recursos e outra de serviços, onde o recurso é utilizado para se obter uma maior acessibilidade, como bengalas, materiais protéticos, roupas adaptadas, softwares, hardwares especiais, etc. Já a parte de serviço é algo disponibilizado profissionalmente ao deficiente, a fim de obter ou usar uma TA, como experimentação e avaliações.

Neste sentido, o objetivo geral deste artigo é apresentar como solução para o problema exposto, o desenvolvimento e implementação de uma bengala automatizada com a tecnologia arduino, sendo capaz de promover auxílio ao deficiente visual através de sensores que, ao identificarem obstáculos no caminho, emitam alarmes sonoros, para que os deficientes visuais que a utilizem possam identificar objetos durante sua locomoção. Compõe os objetivos específicos: (i) promover maior acessibilidade e inclusão social aos deficientes visuais; (ii) prevenir que os cegos não sofram acidentes graves ou mínimas lesões, decorrentes de má identificação de objetos a sua frente em 
seu cotidiano; (iii) promover maior segurança e eficácia durante a locomoção dos usuários e (iv) desenvolver uma bengala automatizada com tecnologia segura e um preço acessível.

Para avaliar a viabilidade e comprovar a eficiência da bengala, foram realizados entrevistas e testes com os deficientes visuais do Centro Municipal de Educação Especial Professora Isoldi Storck. Esta pesquisa de campo forneceu informações e dados relevantes para nortear a construção de um protótipo eficiente e seguro para atender as necessidades dos deficientes visuais.

\section{Materiais e Métodos}

\subsection{Arduino}

O arduino é uma plataforma de criação de protótipos e tecnologias simples em OpenSource (Código Aberto), que traz a possibilidade de pessoas sem capacitação em eletrônica ou programação, criar projetos de maneira simples, com pouca complexibilidade e com um baixo orçamento financeiro [Braga 2002], [Cavalcante et al. 2011].

Tal tecnologia fora desenvolvida no Interaction Design Institute na cidade de Ivrea, na Itália, arquitetado por um grupo de pesquisadores composto por David Cuartielles, Tom Igoe, Gianluca Martino, David Mellis e o criador do projeto Massimo Banzi, no ano de 2005. O objetivo dos pesquisadores era originar um dispositivo com capacidade de fácil programação que também apresentasse um custo acessível, para que pudesse ser utilizado por projetistas amadores, tecnólogos iniciantes e estudantes de Design Tecnológico. Com o objetivo de diversos públicos poderem utilizar o arduino, foi-se adotado o conceito de hardware livre, com intuito de qualquer pessoa o programar, o modelar e personaliza-lo da maneira que desejar [Cavalcante et al. 2011], [Souza et al. 2011).

$\mathrm{O}$ arduino pode ser descrito sucintamente como uma plataforma computacional com estrutura de placa única, constituída por um Microcontrolador central, onde sua estrutura básica é formada por um Microprocessador Atmel AVR e conjuntos de entrada e saída embutidos, funcionando a partir de softwares que podem ser programados para efetuar e realizar operações desejadas [Mcroberts 2010].

Para programar o arduino, a fim de que este funcione e desempenhe as funções desejadas, é utilizado o IDE do arduino, um software livre, no qual apresenta uma linguagem de programação com origem baseada em linguagem $\mathrm{C} / \mathrm{C}++$, que dispõe de grande flexibilidade e compatibilidade para interagir com outras linguagens de tipo C, e por ser uma linguagem completa, oferece uma ótima manipulação [Schildt 1997]. O IDE possibilita a escrita de um código computacional, que servirá como instruções passo a passo do funcionamento do hardware de arduino. Seguidamente, é feito upload para o arduino, onde este executará essas instruções, interagindo com o que estiver conectado a ele [Mcroberts 2010].

Comumente, o arduino está presente em protótipos, engenhos, robótica, designer tecnológico, cursos e trabalhos de automação, utilizado por uma diversidade de pessoas que desejem manuseá-lo. Citando a variedade de áreas que essa tecnologia pode ser 
aplicada, é evidente que o arduino dispõe, de forma prática e facilitada, um acervo de inúmeros possíveis projetos e usos.

\subsection{Revisão Bibliográfica}

O problema da deficiência visual foi e continua sendo tema de estudo em diversos trabalhos científicos, juntamente com o desenvolvimento de uma tecnologia economicamente viável a fim melhorar o estilo de vida das pessoas as quais sofrem dessa deficiência [Hernandes et. al. 2016], [Freitas et al. 2016], [Alves et al. 2014], [Hoefer 2011], [Oliveira 2015]. Tais temáticas tornaram-se algo estudado com grande afinco hodiernamente, e a partir disto, é possível encontrar variados trabalhos que tematizam a construção de tecnologias baseadas em arduino a fim de facilitar a locomoção de deficientes visuais, onde, apresentam métodos seguros, acessíveis e com bons resultados, os quais servem como base teórica para o vigente trabalho.

Em seu trabalho, Hernandes et al. (2015) propõe a criação de uma bengala automatizada que possui um sistema de sensores capazes de detectar obstáculos em volta do deficiente visual e um sistema de alarme com função de alertar a presença desses obstáculos ao indivíduo. Na parte do sensoriamento, os pesquisadores utilizaram sensores ultrassônicos acoplados a bengala em duas posições: um na base, para detectar objetos próximos ao solo, e um no topo, a fim de detectar objetos no plano superior, que possam colidir com a cabeça do indivíduo. Os sensores enviam as informações obtidas a um microcontrolador, por meio da plataforma arduino, que por sua vez envia sinais aos motores de vibração, os quais ficam localizados onde os deficientes seguram a bengala. E por fim, os motores de vibração vibrarão quando os sensores detectarem algum objeto no seu raio de alcance.

Pesquisadores do Departamento de Informática do Instituto Federal do Sertão de Pernambuco trabalharam em um projeto similar. Apesar deste protótipo seguir a mesma estrutura com arduino do protótipo anteriormente citado, ele se difere por apresentar a possibilidade de o protótipo interagir com smartphones via bluetouth, com o objetivo de utilizar os sensores do próprio smartphone. O método apresentado trás o incremento do módulo de bluetouth RS232 HC-05, o qual possibilita o envio de informações ao celular e também a utilização de um aplicativo para Android, desenvolvida na plataforma MIT AppInventor 2. Este aplicativo irá interagir com os sensores do celular, conectando-se com o arduino via bluetouth [Freitas et al. 2016].

Também há projetos nessa área que utilizam redes neurais, as quais ajudam o usuário a se adaptar a velocidade de detecção de objetos da bengala, onde quanto mais rápido o deficiente visual caminhar, mais rápido os objetos a sua frente serão detectados. Esse algoritmo de rede neural é executado de forma constante por conta da possibilidade de variação na velocidade de caminhada do usuário. Os sensores utilizados neste projeto dispõem recursos de aprendizagem por reforço, em que, os sensores ultrassônicos, assim como nos outros projetos, também se posicionam na parte inferior e superior da bengala [Alves et al. 2014].

A Grathio Labs também desenvolve estudos com objetivo de auxiliar deficientes visuais a partir do uso da tecnologia arduino. O projeto Tacit faz parte de seu acervo de estudos, onde, o mesmo consiste em fazer uso de luvas com sensores sonares que podem identificar objetos em distâncias de 2,5 centímetros até 3 metros, e traduzir esta informação em forma de pressão por meio do pulso do deficiente visual. Esse projeto é 
em código aberto, o que torna possível sua utilização pública e até mesmo modificações no mesmo [Hoefer 2011].

Semelhantemente aos projetos que lidam com as bengalas automatizadas, também foi desenvolvida uma Boina Digital. Esta possui o mesmo sistema da bengala automatizada, inclusive o uso do arduino para a mesma finalidade, porém seus sensores só podem identificar obstáculos que estão à altura da cabeça [Oliveira 2015].

Todos os trabalhos anteriormente revisados apresentam resultados positivos, levando-se a crer que o desenvolvimento e a construção de uma bengala automatizada com uso da tecnologia arduino é possível, e que está é apta para promover auxílio e melhoria de vida a pessoas que possuem deficiência visual.

\subsection{Materiais Utilizados}

Para a montagem da Bengala Automatizada foram utilizados diversos materiais, tanto ferramentas arduino, quanto materiais externos, os quais serão listados e descritos na Tabela 1.

\subsection{Pesquisa de Campo}

Durante a elaboração do projeto, a presente equipe realizou uma pesquisa de campo no Centro Municipal de Educação Especial Prof. Isoldi Storck, que presta serviços a deficientes visuais e auditivos. A referida pesquisa teve como principal objetivo coletar os dados que nortearam a criação de uma tecnologia sensorial capaz de promover maior acessibilidade aos cegos.

A coleta de dados ocorreu por meio de pesquisa quantitativa e pesquisa qualitativa, onde, apesar do pequeno número de entrevistados, a pesquisa quantitativa buscou analisar, a partir de gráficos, o índice da falta de acessibilidade, a viabilidade da criação da bengala automatizada e sua avaliação quando montada. Já a pesquisa qualitativa visou colher os principais atributos que deveriam estar presentes na bengala automatizada e, seguidamente, as possíveis melhorias que deveriam ser feitas na mesma.

A pesquisa de campo foi dividida em duas etapas, onde, ambas foram realizadas a partir do emprego de questionários. A primeira ocorreu antes da criação da bengala e a segunda etapa cumpriu-se após a montagem da mesma.

A primeira etapa possui o intuito de compreender melhor as necessidades dos alunos cegos e comprovar a necessidade e viabilidade da criação de uma bengala automatizada. Nesta visita ao centro de educação em questão, foram consultados um número de quatro alunos, onde estes responderam às perguntas abaixo:

- Pergunta 1: Sofre com a falta de acessibilidade no seu cotidiano?

- Pergunta 2: Quão frequente sofre acidentes ao esbarrar em objetos?

- Pergunta 3: Quais são as maiores dificuldades que enfrenta?

- Pergunta 4: Já teve contato com algum projeto semelhante a este?

- Pergunta 5: Pensa que uma tecnologia sensorial como está o ajudaria em sua locomoção?

Após o questionário da primeira etapa ser respondido, com as respostas obtidas foram realizadas as implementações e as automações necessárias na bengala. Posteriormente, quando finalizada a montagem, a equipe executora retornou à 
instituição a fim de realizar a segunda etapa da pesquisa de campo, com o objetivo de testar o protótipo, comprovar se o mesmo desempenhava as funções inicialmente esperadas e ver a avaliação que os alunos dariam a mesma.

Tabela 1 - Lista de materiais utilizados

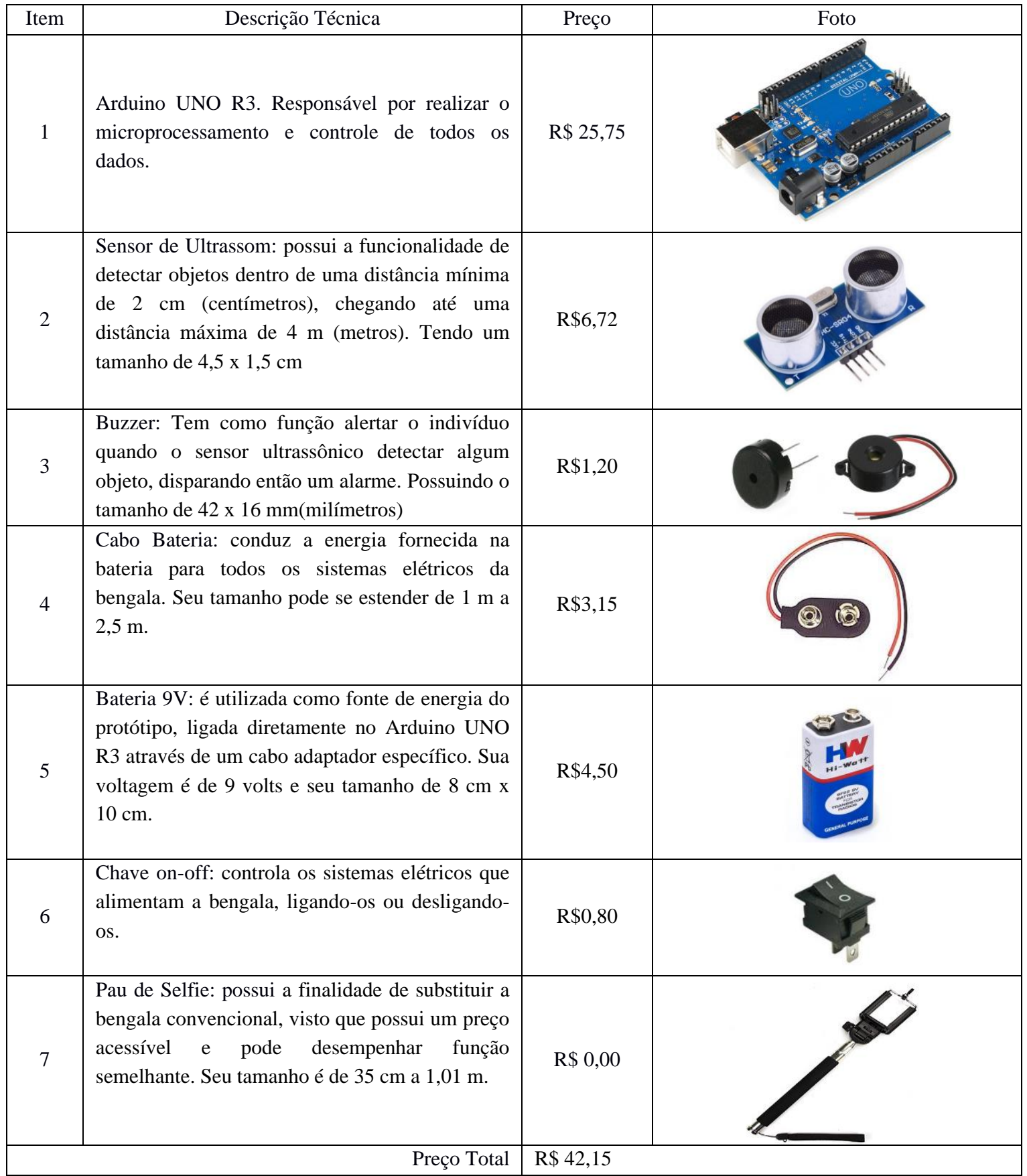

Nesta etapa, foi feita as seguintes perguntas:

- Pergunta 1: O sistema sensorial lhe proporcionou o auxílio em sua locomoção?

- Pergunta 2: As distâncias escolhidas para soar as notificações de proximidade são eficazes?

- Pergunta 3: Você utilizaria uma bengala com este tipo de tecnologia em seu cotidiano? 
- Pergunta 4: Qual é sua avaliação para este protótipo?

- Pergunta 5: Quais são as recomendações que deseja fazer a fim de melhorar o protótipo?

Logo que obtidos os resultados da segunda fase da pesquisa de campo foi possível compreender a importância da criação de uma tecnologia sensorial automatizada capaz de promover maior acessibilidade aos cegos, visto que, os mesmos sofrem constantemente em seu cotidiano por dificuldades que a bengala automatizada se vê capaz de sanar.

\subsection{Montagem do Protótipo}

Para a construção do projeto, foi utilizado materiais de baixo custo e fácil aquisição no mercado, proporcionando um protótipo simples e barato, mas que atende perfeitamente as expectativas de seu uso. Utilizou-se de um pau de selfie de alumínio de aproximadamente 1 metro para a bengala auxiliar, substituindo assim a bengala convencional própria para cegos.

No topo deste pau de selfie fora implementada a chave On-Off, para que o usuário possa ligar ou desligar a bengala facilmente. Logo abaixo, na região onde posiciona-se a mão que irá guiar a bengala, foi criado um pequeno relevo que indica ao cego qual será a região da bengala que deverá ser posicionada para frente. Seguidamente, foram acoplados sensores ultrassônicos nas extremidades superiores e inferiores, com ambos fazendo leitura dos objetos entre um ângulo de $90^{\circ}$ e uma distância programada de $2 \mathrm{~m}$. A figura a seguir ilustra este processo.

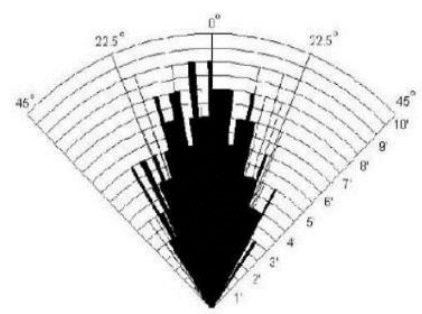

Figura 2. Distribuição de ângulos captada pelos sensores ultrassônicos.

O sensor ultrassônico fixado no plano inferior possui a função de detectar objetos próximos ao solo, como degraus, objetos e animais que porventura, estejam no caminho do deficiente, enquanto o sensor ultrassônico superior é responsável por detectar objetos próximos a cabeça que possam não ser detectadas pelo sensor inferior, como orelhões, caçambas de lixo e galhos de árvores.

Abaixo do sensor ultrassônico superior, foram implementados o microcontrolador arduino UNO R3, o buzzer e logo abaixo, a bateria 9V, para alimentar energicamente todo o sistema. Logo, tais componentes foram fixados ao pau de selfie com auxílio de solda e fita adesiva, sendo conectados entre si a partir de fios elétricos externos e internos, os quais passam por dentro da bengala conectando as extremidades até a central de controle a fim de garantir segurança e um bom design ao protótipo.

A lógica de atuação realizada pelo arduino UNO-R3 habilita o envio de sinais ao Buzzer de maneiras distintas, conforme a distância em que o obstáculo se encontra dos sensores. Desta maneira, quanto mais próximo o objeto se encontrar do sensor, mais 
intenso e repetitivo será o sinal de alerta emitido, com os intervalos de distâncias definidos via programação.

O modo de funcionamento do protótipo é relativamente simples, onde os sensores fazem uma varredura do local a ser percorrido e enviam as informações para o microcontrolador arduino UNO-R3, que por sua vez é responsável por definir os limites de percepção dos sensores e a intensidade do sinal de alerta, de acordo com a iminência de uma colisão. Recebido as informações do local, o microcontrolador, então, controlará de acordo com a proximidade de objetos, a sonoridade emitida pelo Buzzer, a qual alertará o usuário acerca da distância em que o mesmo se encontra de um possível obstáculo. Todo esse sistema só entra em funcionamento com a energia fornecida pela bateria, onde, será acionada a partir dos comandos provenientes da chave on-off.

A programação do circuito elétrico foi realizada, inicialmente, numa placa protoboard, onde foi possível montar e programar os circuitos sem ter a necessidade de soldar os componentes, para assim, evitar possíveis erros durante a fixação dos componentes na bengala. Desta forma, o esquema elétrico implementado pode ser exemplificado na Figura 3, onde, os sensores que estão conectados ao microcontrolador UNO-R3, enviam informações ao mesmo, que posteriormente, após processá-las, encaminha os possíveis comandos ao alarme Buzzer.

A programação dos componentes do protótipo fora feita a partir a plataforma IDE do arduino, a qual é habilitada e direcionada a realizar a programação de componentes arduinos.

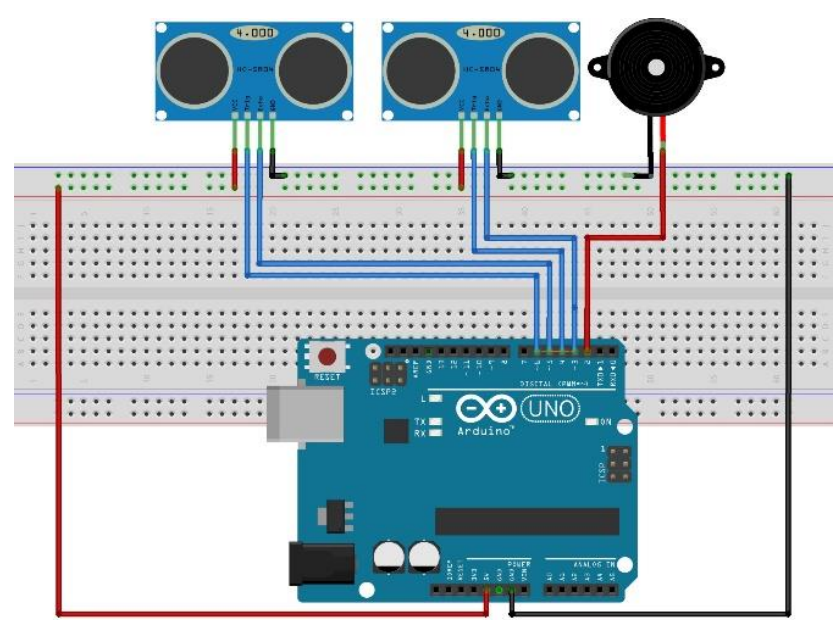

Figura 3. Circuito elétrico da bengala automatizada.

\section{Resultados e Discussões}

Após a montagem da bengala automatizada ser concluída, foram realizados testes entre a equipe desenvolvedora a fim de comprovar a funcionalidade da mesma. A partir disso, obteve-se como resultado do presente trabalho um protótipo de bengala automatizada, devidamente montado e programado, no qual desempenhava as funções esperadas inicialmente, de maneira segura e eficaz, apresentando um comprimento de $1,5 \mathrm{~m}$ e espessura variando de $10 \mathrm{~cm}$ nas regiões com mais componentes a $3 \mathrm{~cm}$ nas regiões com menos componentes. 
Eficientemente, a bengala automatizada é capaz de detectar os objetos em seu raio de alcance a partir da análise de comprimento de ondas realizado pelos sensores superiores e inferiores, e enviar essas informações ao seu microcontrolador Arduino UNO R3, que seguidamente, orienta o buzzer a emitir alertas sonoros conforme a distância que o obstáculo se encontra dos sensores, a fim de conscientizar o usuário a respeito da proximidade entre ele e o objeto. A Figura 4 ilustra o protótipo montado.

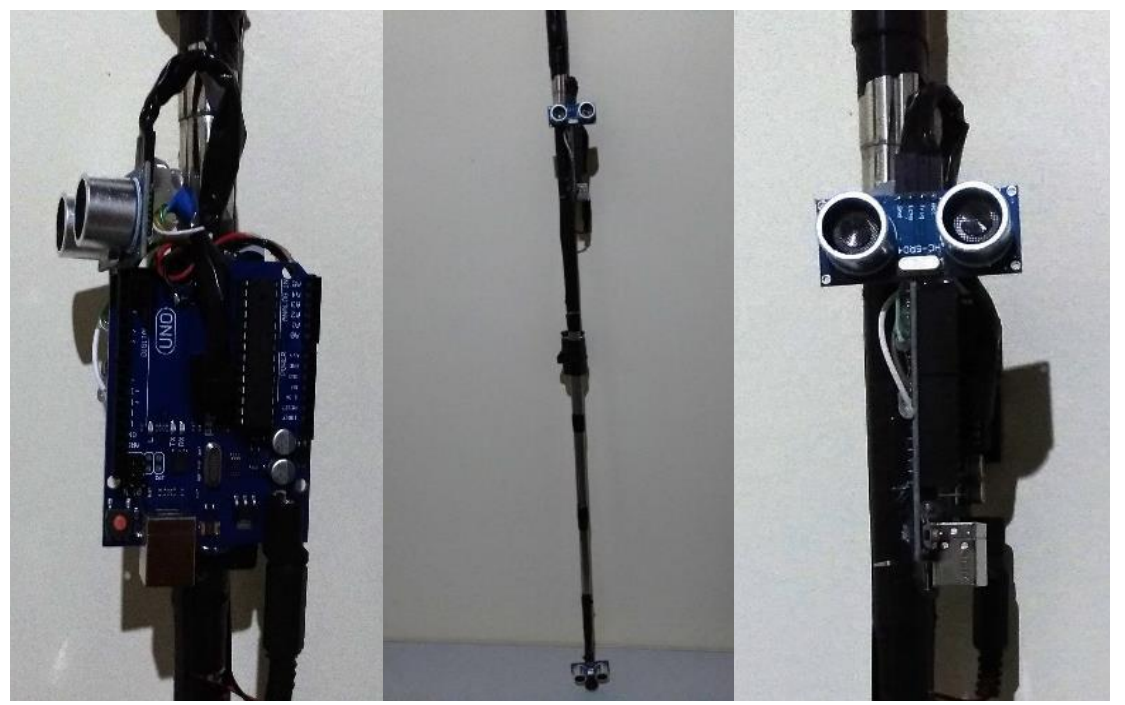

Figura 4. Protótipo de bengala automatizada pronto.

O protótipo de bengala automatizada apresenta quatro espécies de alertas, onde iniciam-se quando o sensor identifica algum objeto à $2 \mathrm{~m}$ de distância, o levando a emitir alertas sonoros mais amenos, com repetições moderadas. Quando o usuário se aproxima do obstáculo e encontra-se à uma distância de $80 \mathrm{~cm}$ do mesmo, esse alerta passa a se repetir com um menor intervalo de tempo, ao passo que, ao usuário atingir a distância de $50 \mathrm{~cm}$ do objeto em questão, se aumentará a intensidade e as repetições seguidas do alerta sonoro. Logo, no momento em que o obstáculo estiver a $30 \mathrm{~cm}$ do usuário da bengala, a mesma emitirá alertas muito frequentes e intensos, para conscientizar a extrema proximidade do objeto, a fim do usuário desviar do obstáculo, evitando possíveis colisões.

\subsection{Resultados da Pesquisa de Campo}

Durante a primeira fase da pesquisa de campo realizada no Centro Municipal de Educação Especial Prof. Isoldi Storck, com a aplicação do questionário, obteve-se os resultados apresentados na figura 5.

Como ilustra o gráfico A, todos os deficientes visuais alegaram sofrer falta de acessibilidade, pois, segundo eles a sociedade carece de meios suficientes para lidarem com deficiências como está, principalmente os ambientes públicos. No gráfico $\mathrm{B}$, um dos deficientes alegou não sofrer grandemente colisões com objetos, pois, por ser cego de nascença, já está melhor adaptado a sua deficiência em relação aos colegas, que apresentaram cegueira ao longo do tempo. No gráfico C, é possível ver o baixo contato dessa comunidade com projetos voltados a promover auxílio a deficientes visuais, pois 
pesquisas e projetos que visam desenvolver tecnologias relacionadas à acessibilidade são pouco fomentadas e consequentemente pouco realizados. Seguidamente, no gráfico $\mathrm{D}$, pode-se comprovar que, de acordo com os alunos deficientes visuais, a perspectiva da criação de uma bengala com tecnologia sensorial seria de grande auxílio em seu cotidiano.
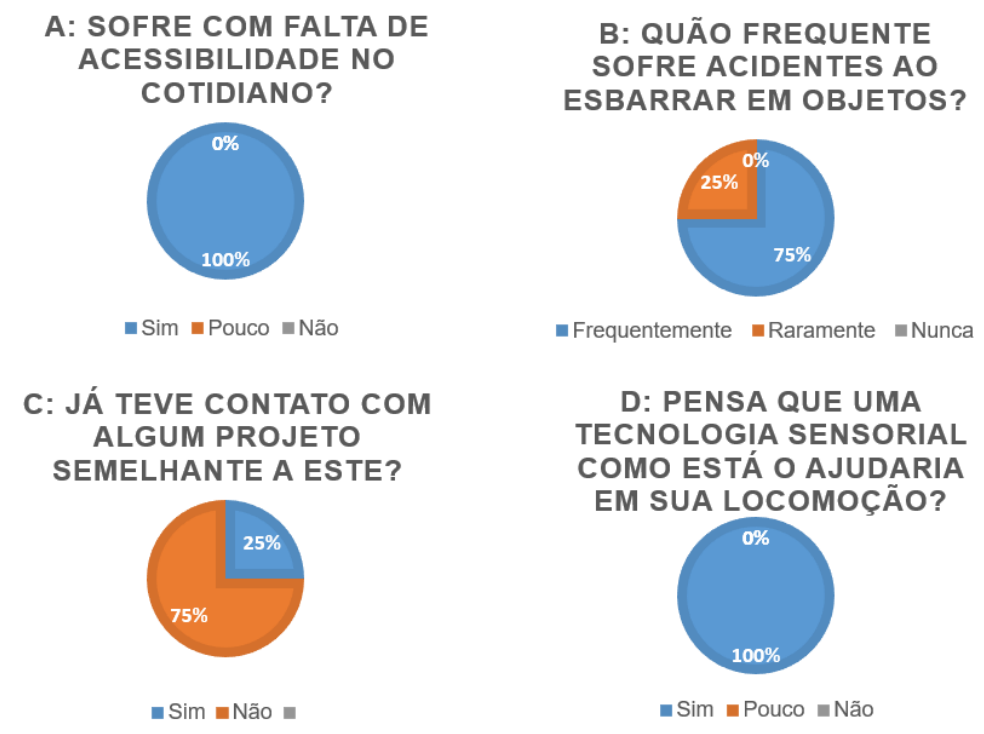

Figura 5. Primeira etapa da pesquisa de campo.

Quando questionados sobre os principais critérios que a bengala deveria possuir, os alunos recomendaram que a mesma possuísse um preço acessível, que fosse leve e de fácil manuseio.

Posteriormente, após a bengala automatizada estar finalizada, foi realizada a segunda etapa da pesquisa de campo, onde a equipe executora retornou ao Centro de Educação Especial Prof. Isoldi Storck com o objetivo de testar a bengala com os alunos cegos da instituição, para observar como os mesmos se adaptaram ao objeto. Primeiro, alunos foram instruídos pela equipe executora a como utilizar a bengala e logo, os mesmos a manusearam sozinhos e andavam pelo local, desviando dos objetos à sua frente.

Seguidamente, foi aplicado o segundo questionário de perguntas, referente a experiência que tiveram ao utilizarem a bengala automatizada. A partir da aplicação do questionário, foi possível obter os seguintes dados apresentados na figura 7.

Como é possível observar na figura 7 , os gráficos $\mathrm{E}$ e $\mathrm{F}$ comprovam que a bengala automatizada atingiu seu objetivo de auxiliar, de maneira segura, a locomoção dos cegos em seu cotidiano por meio de seu sistema sensorial e de alerta.

A partir do gráfico $\mathrm{G}$, evidencia-se que a bengala automatizada se mostra viável e útil aos deficientes visuais, tendo em vista que, unanimemente, os consultados desejaram fazer uso desta tecnologia em seu cotidiano. No gráfico $\mathrm{H}$, de maneira majoritária, os alunos classificaram a bengala como boa, pois de acordo com eles, por ainda ser um protótipo a mesma ainda poderia apresentar pequenas melhoras em seu tamanho e peso. 


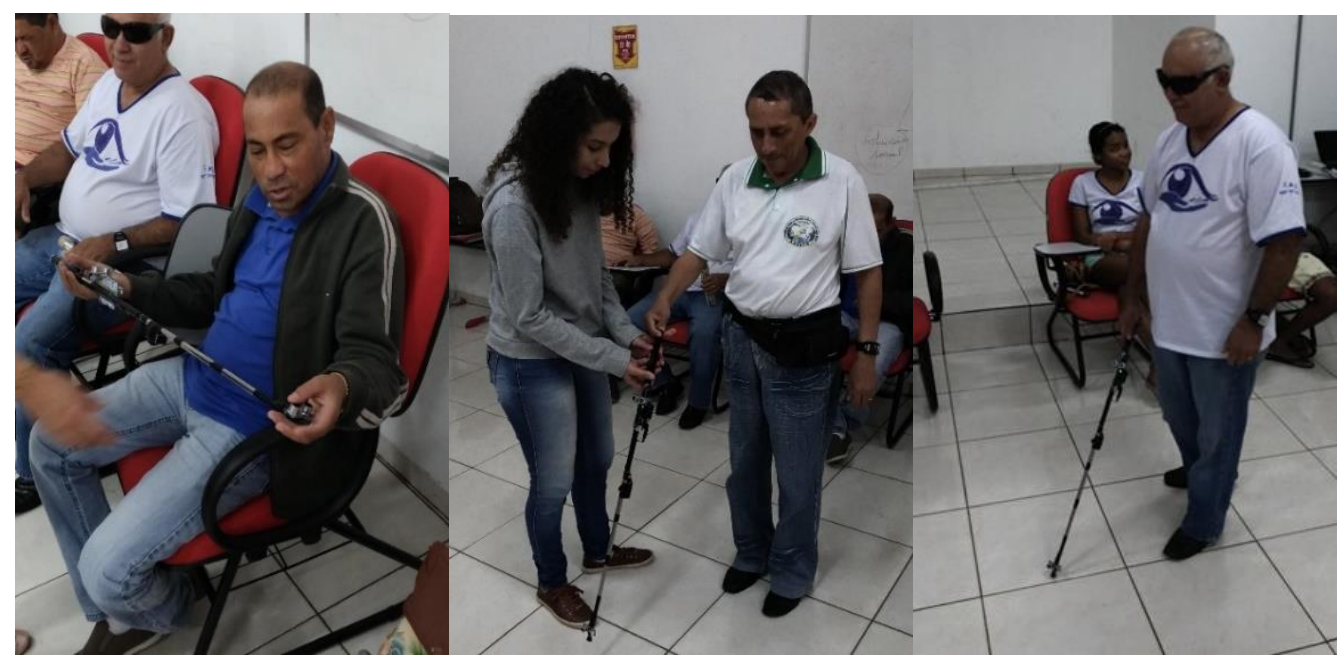

Figura 6. Relato da experiência no teste do protótipo.

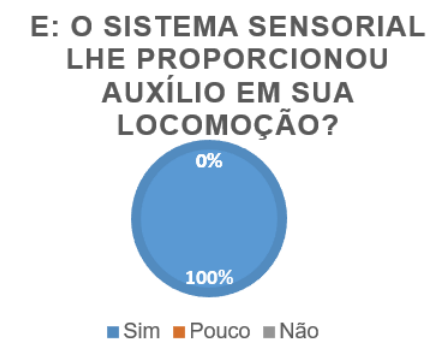

G: VOCÊ UTILIZARIA UMA BENGALA COM ESTE TIPO DE TECNOLOGIA EM SEU COTIDIANO?

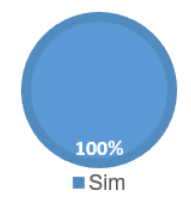

F: AS DISTÂNCIAS ESCOLHIDAS PARA SOAREM AS NOTIFICAÇÕES SÃO EFICAZES?

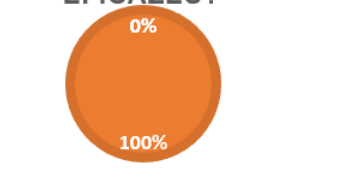

•Não, deviam ser menores - Sim

H: QUAL É SUA AVALIAÇÃO PARA O PRODUTO?

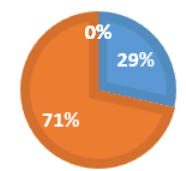

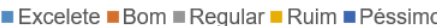

Figura 7. Segunda etapa da pesquisa de campo.

Após finalizada a pesquisa de campo, ao analisar os dados obtidos foi possível concluir que os objetivos inicialmente esperados foram cumpridos, visto que a bengala desempenhou suas funções esperadas e atendeu as expectativas da comunidade externa consultada de maneira segura e eficiente.

\subsection{Discussões}

$\mathrm{Na}$ parte qualitativa da pesquisa, quando questionados sobre quais eram as recomendações que desejavam fazer, os mesmos apontaram que o protótipo deveria ter um comprimento maior, pois seu tamanho estava pequeno para uso. Também alegaram que o protótipo deveria ser um pouco mais leve, pois, com o tempo segurando-a, o braço fica cansado e dificulta sua utilização. Corroboraram também ao sugerir que a mesma fosse dobrável, pois desta maneira facilita seu porte quando a mesma não estiver sendo utilizada. Seguidamente, indicaram a implementação de um roller na ponta da bengala, para que não precisassem batê-la no chão, mas sim rola-la. 
Perante esta análise, foi possível observar que mesmo cumprindo a função de detectar os objetos a frente e alertar os usuários, pode-se implementar as citadas melhorias na bengala automatizada, a fim de tornar mais confortável o seu uso aos deficientes visuais.

\section{Conclusões}

Perante o grande número de casos de cegueira no Brasil e no mundo, o referido trabalho discorreu a respeito do desenvolvimento de uma bengala automatizada com tecnologia arduino, a qual atua como tecnologia assistiva para com deficientes visuais. Esta bengala possui a capacidade de identificar obstáculos em um raio de $2 \mathrm{~m}$, com um ângulo de $90^{\circ}$, onde, quando os identifica, emite um alerta auditivo para que o usuário tenha ciência da proximidade do obstáculo.

Isso só é possível por haver sensores ultrassônicos acoplados à extremidade superior e inferior da bengala, que são capazes de identificar, através de ondas sonoras, os objetos em seu raio de alcance, e, quando os identifica, envia essas informações ao micro-controlador arduino UNO R3, o qual processa essas informações e encaminha comandos ao buzzer, que é acionado, emitindo alertas sonoros conforme a distância que o objeto está, em relação a bengala.

A realização deste trabalho contou com o apoio do Centro de Educação Especial Prof. Isoldi Storck, que disponibilizou os alunos cegos da instituição a responderem os questionários presentes no projeto e realizarem os testes e avaliações da bengala automatizada.

Logo, foi possível comprovar que a bengala automatizada, após finalizada, é uma tecnologia segura, eficiente, inovadora, e de grande utilidade para a deficientes visuais, pois cumpre com suas funções desejadas. Todavia, a mesma pode ser melhorada com implementação de um roller, tornando-a mais leve, maior e dobrável.

A idealização do presente projeto não só trouxe aos discentes envolvidos conhecimento acerca de robótica e lógica de programação, como também fomentou a criação de pesquisas no âmbito social da região, com ênfase no desenvolvimento de tecnologia voltada a acessibilidade, o que despertou nos alunos a empatia de entender a importância de utilizar os avanços tecnológicos em prol da melhor adaptação de deficientes no meio social.

\section{Referências}

Almeida, A. R. et al. Construção de uma bengala eletrônica para deficiente visual. Revista Interação, v. 18, n. 3, p. 135-153, 2016.

Alves, Franciele AS; Neumann, Alexandre MM; GOUVÊA JR, Maury M. Bengala Inteligente Neural Baseada em Aprendizagem por Reforço para Deficientes Visuais. Encontro Nacional de Inteligência Artificial e Computacional, São Carlos, 2014 p.4.

Braga, N. C. Robotics, Mechatronics, and Artificial Intelligence: Experimental circuit blocks for designers. Woburn, MA, USA, 2002.

Bersche, Rita; Tonolli, J. Carlos. Introdução ao Conceito de Tecnologia Assistiva e Modelos de Abordagem da Deficiência. 2006. Disponível em: <http:/www.bengalalegal.com/tecnologiaassistiva>. Acesso em: 25 abril. 2019.

Brasil. Lei No 11.126, de 27 de junho de 2005. Direito do portador de deficiência visual de ingressar e permanecer em ambientes de uso coletivo acompanhado de cão-guia, Brasília, DF, jun. 2005. 
Disponível em: <http:/www.planalto.gov.br/ccivil_03/_ato2004-2006/2005/lei/111126.htm>. Acesso em: 12 abril. 2019.

Brasília DF. Portador de deficiência Visual Guia Legal.3ª .ed. Brasília, 2013.

Bueno, Alessandro Cardozo. Bengala Eletrônica para Deficientes Visuais. 2010. 26f. Trabalho de Conclusão de Curso - Engenharia da Computação, Universidade Positivo, Curitiba, 2010.

Cavalcante, M. A.; Tavalaro, C. R. C.; Molisani, E. Física com Arduino para iniciantes. Revista Brasileira de Ensino de Física. v. 33, n. 4, p. 4503-9, 2011.

Freitas, Alice; Lima, Atos; Santos, Marcelo. Protótipo de Bengala Inteligente de Baixo Custo para o Auxílio de Deficientes Visuais. Anais dos Workshops do V Congresso Brasileiro de Informática na Educação, 2016, p. 1344-1353.

Florio, Lucia Helena. Entendendo o que é ACUIDADE VISUAL. 230 pg. 2018.

Hernandes, J.; Aragão, G. F.; Lima, F. P. A. Bengala Automatizada Para Deficientes Visuais. In: XVI Encontro de Ensino, Pesquisa e Extensão (ENPEX), Araçatuba-SP, 2016, pg. 1-8.

Hoefer, Steve. Meet The Tacit Project. It's Sonar For The Blind. Grathio Labs, 2011. Disponível em: $<$ http://grathio.com/2011/08/meet-the-tacit-project-its-sonar-for-the-blind/>. Acesso em 24 set. 2018.

Ibge (Instituto Brasileiro de Geografia e Estatística). Censo Demográfico 2010: Características gerais da população, religião e pessoas com deficiência. Disponível em: $<$ https://biblioteca.ibge.gov.br/pt/biblioteca-catalogo?view=detalhes\&id=794> Acesso em: 01 jul. 2018.

Mcroberts, M. Arduino básico. São Paulo: Novatec, v. 1, 2010.

Oliveira, Carlos Eduardo S. de. Bengala Digital e Boné Digital à Serviço da Pessoa com Deficiência Visual. Instituto Federal de Educação, Ciência e Tecnologia da Bahia, 2015.

Pinto, Pedro. Tecnologias Assistivas no Brasil. Disponível em: $<$ http://www.acessibilidade.net/at/kit2004/Programas\%20CD/ATs/cnotinfor/Relatorio_Inclusiva/pdf/ Tecnologias_assistivas_Br_pt.pdf $>$. Acesso em: 13 jun. 2018.

Ramke, J.; Gilbert, C. E. Universal eye health: are we getting closer? Journal of The Lancet Global Health, United Kingdom, v.5, n.9, p. e843-e844, 2017.

Schildt, Herbert. C Completo e Total $.3^{\mathrm{a}}$.ed. Tradução: Roberto Carlos Mayer. São Paulo: Makron Books do Brasil, 1997. 816 p.

Santos, D. R. de G.; Ferreira, W. R. B.; Borges, M. A.; Gonçalves, R. S. Desenvolvimento de uma bengala eletrônica para locomoção de pessoas com deficiência visual. CONEN, Paraíba, 2010.

Silva, B. S. G. et al. Desenvolvimento de uma bengala eletrônica de baixo custo baseada em sensores ultrassônicos. Mostra Nacional de Robótica (MNR), 2018.

Souza, A. R.; Paixão, A. C.; Uzêda, D. D.; Dias, M. A.; Duarte, S.; Amorin, H. S. A placa Arduino: uma opção de baixo custo para experiências de física assistidas pelo PC. Revista Brasileira de Ensino de Física, v. 33, n. 1, 1702, 2011.

Teixeira, Luzimar. Exame de Visão. Disponível em: <http://www.luzimarteixeira.com.br/wpcontent/uploads/2010/05/tabela-snellen-uso-e-interpretacao.pdf $>$ Acesso em 24 set. 2018.

Temporini, Edméa Rita; KARA-JOSE, Newton. A perda da visão: estratégias de prevenção. Arq. Bras. Oftalmol. São Paulo, v. 67, n. 4, p. 597-601, 2004. 